\title{
MAPEAMENTO DAS INSTITUIÇÕES FEDERAIS DE ENSINO SUPERIOR DA REGIÃO NORDESTE DO BRASIL NA WEB
}

\author{
MAPEAMIENTO DE LAS INSTITUCIONES FEDERALES DE EDUCACIÓN \\ SUPERIOR DE LA REGIÓN NORESTE DE BRASIL EN LA WEB
}

\author{
Nadia Vanti - nvanti@hotmail.com \\ Doutora em Comunicação e Informação \\ Docente da Universidade Federal do Rio Grande do Norte (UFRN).
}

\section{Resumo}

Como parte de uma pesquisa mais ampla a respeito da presença das instituições federais de ensino superior brasileiras na Web, realizou-se uma análise das universidades da região Nordeste do país por meio da aplicação de diferentes indicadores webométricos: tamanho do sítio, visibilidade, fator de impacto Web - FIW, luminosidade e densidade da rede. Para a medição do tamanho dos sítios Web e de sua visibilidade no mundo virtual, utilizou-se o motor de busca Yahoo e o fator de impacto foi calculado por meio de uma fórmula especialmente criada para este fim. A luminosidade destes sítios foi calculada tendo como ferramenta o programa mapeador Xenu Link Sleuth. A densidade da rede foi obtida também com a aplicação de uma fórmula. O programa Ucinet6 foi utilizado para a visualização das conexões entre os sítios das universidades da região. Os resultados demonstraram que as universidades maiores são também aquelas mais acessadas e as menores são as que costumam acessar mais websites. $O$ fator de impacto não apresentou resultados significativos quando aplicado a sítios Web e a densidade da rede das universidades federais da região Nordeste do Brasil mostrou-se muito baixa, o que representa um enorme potencial inexplorado no que diz respeito à conectividade destas instituições com seus pares neste ambiente.

\section{Palavras-chave}

Webometria. Estudos métricos da informação. Avaliação de sítios acadêmicos. IFES Nordeste. 


\section{INTRODUÇÃO}

$\mathrm{Na}$ Sociedade do Conhecimento, a Web tornou-se uma plataforma ou canal de comunicação e de informação privilegiado no âmbito acadêmico. Isto faz com que sejam cada vez mais necessárias a análise e a avaliação das informações que circulam na rede, especialmente daquelas disponibilizadas em sítios Web e as relações que estabelecem com outros sítios neste ambiente.

$\mathrm{O}$ trabalho aqui apresentado faz parte de uma pesquisa mais ampla que abrange a análise webométrica de todas as Instituições Federais de Ensino Superior do Brasil (IFES). O seu desenvolvimento justifica-se pela crescente disponibilização de informações de toda natureza na Web e o ainda tímido desenvolvimento de pesquisas no Brasil que objetivem medir a presença das informações acadêmicas neste ambiente nos moldes daquelas que são realizadas para avaliar as informações impressas por meio da Bibliometria e Cientometria, tendo como fonte de dados o Institut for Scientific Information - ISI.

No Brasil, apenas em 2002 começaram a serem produzidas as primeiras pesquisas na área da Webometria (VANTI, 2002). Entretanto, somente a partir de 2005 iniciaram as pesquisas tendo como tema central a análise das redes sociais entre sítios Web, por meio de hiperlinks (VANTI, 2005; GOUVEIA, 2007). Fato este que contrasta com o que acontece no âmbito internacional, onde há grande interesse por estes temas e já são várias as pesquisas realizadas e vários também os trabalhos publicados (ALMING; INGWERSEN, 1997, THELLWAL, 2002a; 2002b; THELWALL; SMITH, 2002; AGUILLO; GRANADINO; LLAMAS, 2005; AGUILLO; GRANADINO; ORTEGA, 2006; AGUILLO et al, 2007) entre outros.

Neste sentido, Alming e Ingwersen (1997) foram os primeiros pesquisadores a utilizarem a expressão Webometria para se referir aos estudos métricos da informação disponibilizada na Web. Thelwall, entretanto, avançou na explicação deste mesmo termo afirmando que "a razão de ser do termo Webometria seria o de denotar sua origem na Bibliometria e na Informetria e ainda destacar uma perspectiva da Ciência da Informação para os estudos da Web" (2003, p. 3, tradução nossa). Björneborn, mais recentemente, definiu a Webometria como "o estudo dos aspectos quantitativos da construção e uso dos recursos de informação, estruturas e tecnologias na Web, utilizando enfoques bibliométricos e informétricos" (2004, p. 12, tradução nossa). 
Alguns pesquisadores vêm desenvolvendo investigações semelhantes a que se pretende expor neste trabalho, voltadas para a análise dos sítios de universidades de diversos países e continentes. Thelwal (2002a, 2002b), por exemplo, em um primeiro artigo a respeito do assunto, apresentou o desenvolvimento de uma metodologia para analisar os padrões de interlinkagem entre os sítios das universidades britânicas, salientando que a distância geográfica das universidades influencia de forma determinante nos links entre os seus sítios. No segundo trabalho abordou com grande nível de detalhamento a contabilização de links entre pares de universidades, mostrando evidências de um relacionamento linear importante entre tamanho e qualidade da pesquisa tanto das instituições-fonte quanto das instituições-alvo. $O$ autor propôs que este modelo fosse aplicado a outros sistemas universitários nacionais, com a expectativa de vê-lo confirmado por estudos realizados em outros países, propiciando o aprofundamento da exploração de dados acerca dos links Web acadêmicos. Ainda neste mesmo ano, Thelwall e Smith (2002) publicaram outro artigo enfocando o estudo dos interlinks entre sítios Web de universidades da Ásia e Pacífico.

Aguillo é outro autor que se destaca nos estudos destes temas. Em 2005 publicou, em co-autoria com Granadino, artigo acerca do posicionamento do setor acadêmico iberoamericano na Web (AGUILLO; GRANADINO, 2005). Em 2006, os mesmos autores apresentaram um paper no Workshop on Webometrics, Informerics and Scientometrics \& Seventh Collnet Meeting, Nancy, França, em que realizavam uma análise cibermétrica no universo Web acadêmico brasileiro (AGUILLO; GRANADINO, 2006). E em 2007, Aguillo e outros autores escreveram a respeito dos indicadores Web de atividade científica formal e informal na América Latina. (AGUILLO et al., 2007).

Cabe ressaltar ainda que desde 2004 publica-se duas vezes por ano, em janeiro e julho, um ranking que cobre mais de 17 mil instituições de ensino superior por todo o mundo intitulado Webometrics Ranking of World Universities. A lista final, composta por cerca de 6.000 universidades é elaborada pelo Cybermetrics Lab, um grupo de pesquisa ligado ao Consejo Superior de Investigaciones Científicas - CSIC, a maior instituição pública de pesquisa da Espanha. (ESPANHA, 2009). O indicador utilizado para organizar este ranking é a visibilidade dos sítios das universidades na Web, de acordo com o número de links que recebem de outros sítios. Com relação às universidades brasileiras neste ano de 2009, conforme a lista, a USP apareceu em primeiro lugar, alcançando o $38^{\circ}$ lugar no ranking mundial, a UNICAMP em segundo na $115^{\text {a }}$ posição nesse ranking e a UFSC, terceira no Brasil e 134를 no mundo. (USP, 2009). 
Os indicadores webométricos têm sido utilizados, portanto, para análises de sítios de diferentes domínios e assuntos, incluindo os acadêmicos. São empregados para medir o tamanho dos sítios ou o seu número de páginas e a presença de cada um deles na rede de acordo com a quantidade de inlinks externos determinando, assim, a sua visibilidade. Estes dois indicadores costumam estar estreitamente ligados, mostrando que se o primeiro é alto, o segundo tende a sê-lo também. Da relação entre tais indicadores surge outro indicador muito importante que serve para determinar e comparar o grau de atratividade ou influência dos espaços Web, com base no número de links que recebem tendo em conta o seu tamanho ou número de páginas, o fator de impacto Web - FIW. (SMITH, 2009). Foi Smith (1999) quem estabeleceu os critérios para os valores obtidos por meio deste indicador, da seguinte forma: sítios com alto FIW são aqueles que possuem índices significativamente maiores do que 1, ou seja, em média cada página tem mais do que 1 link externo apontando para ela; sítios com médio FIW possuem índices em torno de 1, e sítios com baixo FIW possuem índices significativamente menores do que 1.

Cumpre lembrar que foi Ingwersen (1998) quem sugeriu a criação de um indicador análogo aquele calculado a partir das bases de dados do Institute for Scientific Information - ISI. Segundo o mesmo autor, entretanto, este indicador deve ser aplicado com cautela. Isto se deve ao fato de que conectar-se à uma página Web nem sempre significa que quem conectou-se o fez porque a julgava importante como costuma ocorrer com a citação em materiais impressos. Pode ter sido por outros motivos como, por exemplo, ter sido incluído em um ranking dos piores sítios acerca de um determinado assunto, ou ainda por fazer parte de um conjunto de recursos Web que devem ser conectados por terem o mesmo status que o sítio, entre outros.

Muitos outros indicadores podem ser aplicados ao universo Web como forma de avaliá-lo. A luminosidade costuma ser empregada para examinar o quanto um sítio se relaciona com seus pares neste ambiente. Ao inserir links a outros sítios, um recurso Web está demonstrando, a princípio, que valoriza os sítios apresentados. Assim como nas citações a outros documentos em materiais impressos, o acesso na Web também pode significar o reconhecimento da importância de outro sítio para o seu.

A densidade da rede, por sua vez, afere o quanto e como uma população se relaciona dentro de um determinado universo, por meio de método muito usado nas ciências sociais, a análise de redes sociais. Este indicador é interessante, pois mostra que 
grau de interação existe entre os elementos que compõem uma rede, sejam estes elementos pessoas, números ou, no caso, sítios Web.

Tendo como base as pesquisas já realizadas neste campo, pretende-se aplicar alguns dos indicadores webométricos já utilizados em outros estudos, aos sítios das instituições federais de ensino superior da região Nordeste do Brasil. Busca-se, assim, testar os mesmos indicadores com um objeto de estudo distinto, a fim de verificar a pertinência ou não do uso dos indicadores webométricos e contribuir para o avanço do conhecimento nesta área.

Considera-se, ademais, que a medição e a análise dos sítios das instituições federais de ensino superior da região Nordeste do Brasil poderão trazer grandes benefícios, uma vez que não só permitirão traçar um diagnóstico da situação em que se encontra cada uma delas, quanto possibilitarão a formulação de políticas que tendam a aumentar a visibilidade e o intercâmbio destas Instituições com suas congêneres da região e das demais regiões do país no âmbito da Web.

\section{METODOLOGIA}

A listagem de todas as instituições federais de ensino superior brasileiras foi encontrada no sítio do Ministério de Educação e Cultura - MEC e o seu Localizador de Recursos Universal - URL correspondente foi pesquisado na Internet por meio do motor de busca Google. Aquelas que correspondem à região Nordeste são as seguintes:

\begin{tabular}{|c|c|}
\hline IFES DA REGIÃO NORDESTE DO BRASIL & URL \\
\hline $\begin{array}{l}\text { Fundação Universidade Federal do Vale do São } \\
\text { Francisco - UNIVASF }\end{array}$ & www.univasf.edu.br \\
\hline Universidade Federal do Recôncavo da Bahia - UFRB & $\underline{w w w . u f r b . e d u . b r}$ \\
\hline Universidade Federal de Alagoas - UFAL & www.ufal.br \\
\hline Universidade Federal da Bahia - UFBA & $\underline{w w w . u f b a . b r}$ \\
\hline Universidade Federal do Ceará - UFC & $\underline{w w w . u f c . b r}$ \\
\hline Fundação Universidade Federal do Maranhão - UFMA & www.ufma.br/ \\
\hline Universidade Federal da Paraíba - UFPB & www.ufpb.br \\
\hline Universidade Federal de Pernambuco - UFPE & www.ufpe.br \\
\hline Universidade Federal Rural de Pernambuco - UFRPE & www.ufrpe.br \\
\hline Fundação Universidade Federal do Piauí - UFPI & www.ufpi.br \\
\hline Universidade Federal do Rio Grande do Norte - UFRN & www.ufrn.br \\
\hline
\end{tabular}




\begin{tabular}{ll} 
Fundação Universidade Federal de Sergipe - UFS & $\underline{\text { www.ufs.br }}$ \\
Universidade Federal de Campina Grande - UFCG & $\underline{\text { www.ufcg.edu.br }}$ \\
Universidade Federal Rural do Semi-Árido - UFERSA & $\underline{\text { www.ufersa.edu.br }}$ \\
\hline
\end{tabular}

Tabela 1 - Sítios web das IFES da região Nordeste do Brasil

Fonte - Elaboração da autora.

Para a análise webométrica dos sítios acima elencados utilizaram-se os seguintes indicadores: tamanho do sítio, visibilidade, fator de impacto Web, luminosidade e densidade da rede. Retomando o que já foi exposto anteriormente, o tamanho do sítio corresponde ao número total de páginas que ele apresenta e deve ser calculado por meio de um motor de busca.

A visibilidade diz respeito ao número de links recebidos, pelo sítio analisado, desconsiderando os autolinks. Este indicador pode ser medido tanto por um motor de busca (Google, Yahoo, Alta Vista, etc.) quanto por meio de um portal de serviços (Outsource Stategies International ou Marktleap Search Engine Marketing Tolls).

O fator de impacto Web indica a proporção de links que uma homepage recebe em relação ao seu tamanho ou número total de páginas. A luminosidade é o indicador que representa o número de links que um sítio inclui nas suas páginas Web a outros sítios e será calculado com base em um programa mapeador. Já a densidade da rede é o indicador que mostra o quanto um conjunto de sítios se relaciona entre si por meio do estabelecimento de seus links.

Levando em conta as definições dos indicadores Web, este estudo se propõe a analisar cada um deles de acordo com o método mais adequado. O tamanho do sítio Web ou o número de páginas que compõem cada sítio foi determinado, portanto, por meio de pesquisa ao motor de busca Yahoo.

O segundo indicador levantado nesta pesquisa foi o de visibilidade e o motor de busca Yahoo foi mais uma vez a ferramenta de auxílio utilizando-se, entretanto, neste caso, a busca avançada.

Ao se dividir o número de links apontando para uma homepage (o segundo indicador analisado) pelo número total de suas páginas (o tamanho do sítio), obtém-se o valor de outro indicador utilizado para medir a importância de um recurso Web, o fator de impacto Web (INGWERSEN, 1997; THELWALL, 2000), de acordo com a fórmula abaixo:

$$
\text { FIW }=\frac{\text { № de páginas que "linkam" determinado site }}{\text { № de páginas do site "linkado" }}
$$


Já para encontrar o número total de outlinks externos que correspondem à luminosidade dos sítios Web e, ao mesmo tempo, identificá-los na rede, lançou-se mão do Xenu Link Sleuth, programa que oferece o mapa completo de todas as conexões que cada sítio estabelece, mediante a digitação do seu endereço. Depois disto, foi realizada, então, uma depuração manual, selecionando-se dentre todas estas conexões apenas aquelas que se caracterizavam por representarem a luminosidade.

Cabe esclarecer, entretanto, que não foi possível mapear duas das quatorze universidades analisadas. Isto ocorre, porque quando são submetidos ao programa mapeador Xenu, alguns sítios redirecionam tal programa para o Java Script, impedindo, assim, o seu mapeamento.

O último indicador pesquisado neste conjunto de sítios foi a densidade da rede. Este, por sua vez, foi calculado dividindo-se o número de links realmente existentes entre os sítios do universo estudado pelo número de links possíveis de serem estabelecidos entre os sítios deste mesmo universo, de acordo com a seguinte equação:

$$
\mathrm{DR}=\frac{\text { links }}{\mathrm{n} .(\mathrm{n}-1)}
$$

\section{RESULTADOS E DISCUSSÃO}

Apresenta-se, abaixo, uma tabela com os indicadores quantitativos obtidos a partir da metodologia descrita no tópico anterior para calcular cada um deles: tamanho, visibilidade, fator de impacto, luminosidade e densidade da rede. Logo em seguida a tabela é interpretada, tendo em conta os maiores valores encontrados por indicador (ressaltados em negrito).

\begin{tabular}{lrr|r|r|r}
\hline \multicolumn{1}{c|}{ IFES } & TAM. JUN/09 & VISIB. & FIW & LUMIN. ${ }^{*}$ & $\begin{array}{c}\text { CONEXÕES } \\
\text { DA REDE }\end{array}$ \\
\hline UFAL & 937.000 & 1.333 & 0,00 & - & - \\
UFBA & 2.210 .000 & 3.604 & 0,00 & - & 2 \\
UFC & $\mathbf{1 7 0 . 0 0 0 . 0 0 0}$ & $\mathbf{4 3 8 9}$ & 0,00 & 14 & - \\
UFCG & 281.000 & 703 & 0,00 & 380 & 2 \\
UFERSA & 24.800 & 116 & 0,00 & $\mathbf{5 2 3}$ & 4 \\
UFMA & 579.000 & 1.393 & 0,00 & 11 & - \\
UFPB & 1.030 .000 & 2.955 & 0,00 & 126 & $\mathbf{5}$ \\
UFPE & 2.590 .000 & 4.369 & 0,00 & 89 & - \\
UFPI & 952.000 & 1.790 & 0,00 & 22 & - \\
UFRB & 36.300 & 118 & 0,00 & 63 & 1 \\
UFRN & 1.930 .000 & 3.413 & 0,02 & 234 & - \\
UFRPE & 339.000 & 858 & 0,00 & 23 & -
\end{tabular}

* Os sítios da UFBA e da UFAL não puderam ser mapeados. 


\begin{tabular}{lrrrrr} 
UFS & 9.130 .000 & 1.390 & 0,00 & 372 & 1 \\
UNIVASF & 9.360 & 241 & 0,03 & 50 & 1 \\
\hline
\end{tabular}

Tabela 2 - IFES da região Nordeste do Brasil na Web por indicador

Fonte - Elaborado pela autora.

Segundo a tabela acima, o primeiro indicador analisado é o tamanho dos sítios (TAM.) e os números mostram que a instituição de ensino superior da região Nordeste que apresenta o maior número de páginas na Web é a UFC, ultrapassando expressivamente a segunda e a terceira colocada, que são a UFS e a UFPE, respectivamente.

Já tendo em vista a terceira coluna da Tabela 3 (VISIB.), que apresenta o indicador visibilidade, a universidade que exibe o maior número de sítios com links externos apontando para ela é, mais uma vez, a UFC, seguida agora da UFBA e da UFPE. Analisando estes dois primeiros indicadores, a visibilidade parece ter relação com o tamanho do sítio e a conseqüente importância que representam estas universidades na região.

Ao se calcular a proporção de links recebidos por uma homepage em relação ao seu número total de páginas, ou seja, o fator de impacto, mais uma vez se constatou que este indicador não apresenta resultados significativos quando aplicado à Web, diferentemente de quando é aplicado a materiais impressos, como revistas, por exemplo.

Neste estudo, especificamente, o que se pode observar é que os sítios que alcançaram os primeiros lugares no ranking deste indicador são os de universidades que possuem um tamanho menor ou um pequeno número de páginas web, o que faz com que o denominador (o número pelo qual se dividem os inlinks externos) seja muito menor do que o dos sítios de universidades consideradas grandes, não significando, exatamente, atratividade.

Já na análise do indicador luminosidade, levando em conta que duas das quatorze instituições federais de ensino superior não puderam ser mapeadas, verifica-se que o sítio da UFERSA é aquele que apresenta o maior número de links a outros sítios na rede (523), seguido da UFCG (380) e da UFS (372), de acordo com a Tabela 5 (LUMIN.). Pode-se dizer que estas universidades são aquelas que mais se conectam a outras instituições na Web, se comparadas às suas semelhantes.

Ainda com relação à luminosidade, levando-se em conta apenas os dois primeiros lugares, é possível afirmar que as universidades com menos páginas Web, ou seja, de 
menor tamanho, são as que tendem a apresentar mais link, porém a UFS, em terceiro lugar, tendo um número expressivo de páginas Web, vem de encontro a essa premissa.

Com relação à densidade da rede das instituições federais de ensino superior da região Nordeste, apresentada na Tabela 5 (CONEXÕES DA REDE), pode-se verificar que a densidade, com apenas 0,08 ou $8 \%$ de conexões (16) do total possível (182). Cabe destacar que, na tabela, aparece apenas o número correspondente aos links emitidos por cada instituição e não recebidos, pois assim não se duplicam os resultados. Por este motivo sete estão em branco. Porém na Figura 1 é possível perceber mais claramente como as conexões são estabelecidas.

Neste sentido, a universidade que estabelece o maior número de conexões na rede de IFES da região Nordeste é a UFPB com sete inter-relações (cinco links apontando para sítios de outras universidades - UFC, UFAL, UFRN, UFPE e UFBA mais dois link recebidos - UFCG e UFERSA), a UFBA, apresentando cinco inter-relações (dois links apontando para outras universidades - UFRB e UNIVASF e três recebidos - UFPB, UFRB e UFERSA) e a UFERSA (com quatro links apontando para outras universidades UFRN, UFCG, UFPB e UFBA), formando assim três agrupamentos (clusters) nesta pequena rede social (Figura 1).

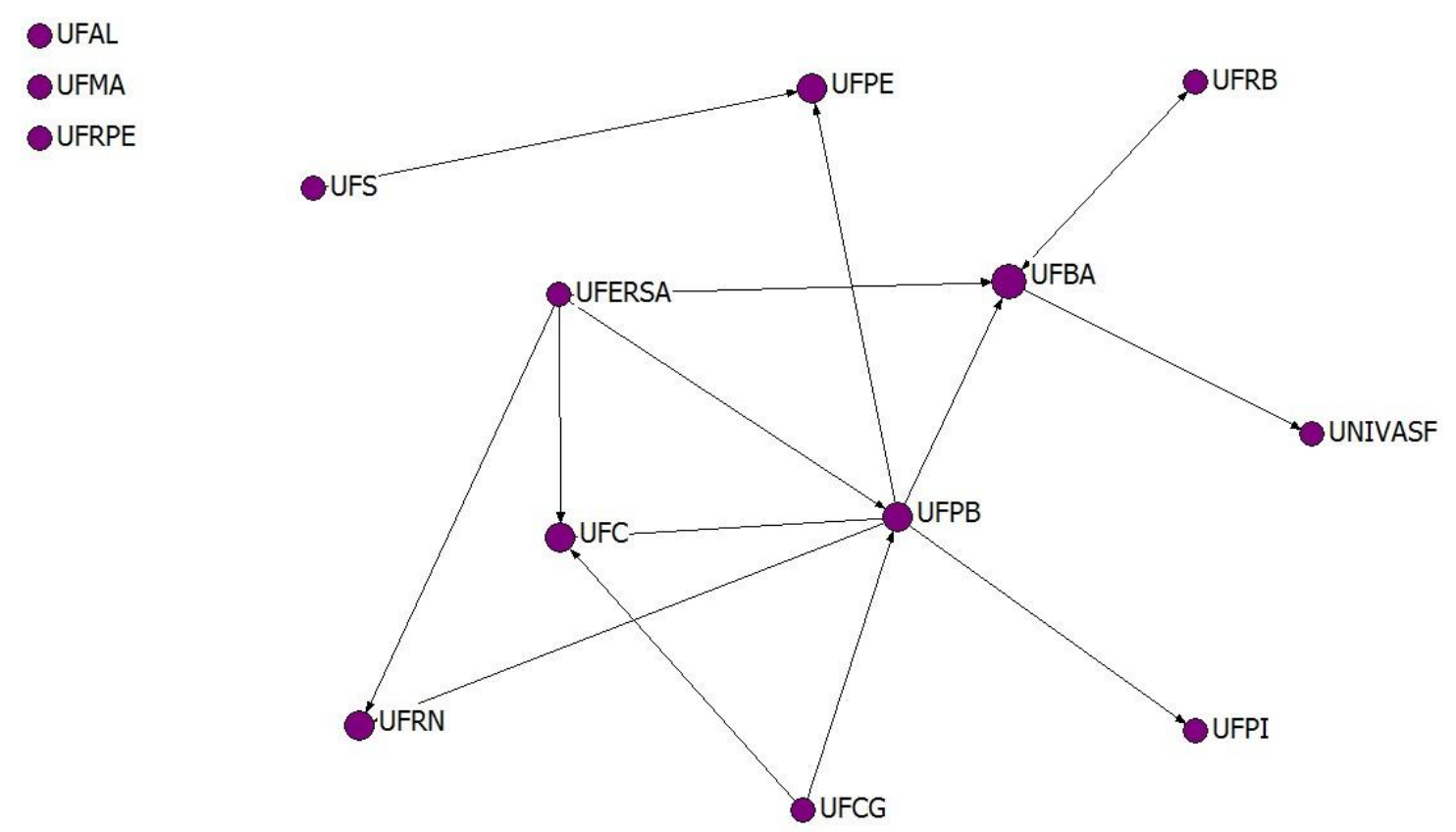

Figura 1 - Conexões entre as IFES da região Nordeste do país na Web.

Fonte - Elaboração das autoras. 


\section{CONCLUSÃO}

A Webometria é um campo novo no âmbito da Ciência da Informação que necessita ainda de muitos estudos teóricos e, especialmente, práticos para a sua consolidação, já que tem como propósito medir as informações em um ambiente bastante instável como é o da Web.

Mesmo levando em conta este fato, foi possível, por meio deste estudo, perceber algumas tendências no que se refere à presença das universidades da região Nordeste na rede.

As universidades que apresentaram um maior número de páginas também foram, na maioria das vezes, aquelas que receberam o maior número de links (ou apresentaram maior visibilidade), possivelmente por serem consideradas mais importantes, mostrando uma forte correlação entre estes dois indicadores. Este é o caso da UFC que aparece em primeiro lugar tanto em tamanho como em visibilidade.

Parece existir também uma correspondência entre visibilidade na Web e "ser citado" na Bibliometria, da mesma forma que entre luminosidade e "citar". Quando se apresentaram os rankings destes dois indicadores, a UFC aparece em primeiro lugar no que diz respeito ao indicador visibilidade e, por outro lado, a UFERSA também em primeiro quando o indicador analisado é a luminosidade, podendo-se arriscar a afirmar que as universidades maiores e mais importantes costumam receber mais links, enquanto que as menores parecem estabelecer conexões a mais com outras instituições.

O indicador fator de impacto, entretanto, mais uma vez demonstrou que, aplicado a sítios da Web, não resulta pertinente, apresentando resultados que não correspondem ao que ocorre normalmente no mundo real. Este indicador parece só ser relevante quando aplicado a material impresso.

Já no que diz respeito à densidade da rede das instituições federais de ensino superior da região Nordeste do país, ela é muito baixa, alcançando menos de $10 \%$ do total de conexões possíveis. O que parece indicar que estas instituições apresentam um enorme potencial inexplorado no que se refere à conectividade entre seus pares no ambiente Web.

De qualquer forma, foi possível verificar que a UFPB é a instituição que mais conexões estabelece com outras universidades, sendo considerada, portanto, a mais "sociável" da região. Este é um típico caso em que os motivos pelos quais ocorre este 
fato deveriam ser investigados mais profundamente. Chama a atenção que mesmo a UFPB sendo uma universidade com um tamanho Web não tão expressivo quanto a UFC, a UFS e a UFPE e também não se distinguindo por ter visibilidade nem luminosidade tão altas, ainda assim é a que mais conexões estabelece nesta rede.

Por fim, cabe salientar que, mesmo com as limitações que possam ter ocorrido nas análises por conta da falta de mapeamento da luminosidade de dois sítios e do próprio método webométrico, ainda em processo de consolidação e aplicado a apenas uma parte do total do universo a ser pesquisado, foi possível traçar o perfil das relações que se formam na Web entre as instituições federais de ensino superior da região Nordeste do Brasil. Também se considera que, de certa forma, este estudo contribuiu para "abrir as portas para o aprofundamento da exploração de dados sobre os links Web acadêmicos", como Thelwall (2002b) sugeriu que fosse feito também em outros países.

\section{REFERÊNCIAS}

ALMIND, T.C.; INGWERSEN, P. Informetric analyses on the world wide web: methodological approaches to "Webometrics". Journal of Documentation, London, v. 53, n. 4, p. 404-26, 1997.

AGUILLO, I.; GRANADINO, B.; LLAMAS, G. Posicionamiento en el web del sector académico iberoamericano. Interciéncia, v. 30, n. 12, p. 735-8, 2005.

AGUILLO, I.; GRANADINO, B.; ORTEGA, J. L. Brazil academic webuniverse revisited: a cybermetric analysis. In: WORKSHOP ON WEBOMETRICS, INFORMETRICS AND SCIENTOMETRICS \& SEVENTH COLLNET MEETING. Nancy, FR, mai. 2006.

AGUILLO et al. Indicadores web de actividad científica formal e informal en Latinoamérica. Revista Española de Documentación Científica, Madrid, v. 30, n. 1, p. 49-60, 2007.

BJÖRNEBORN, Lennart. Small-world structures across an academie web space: a library and information science approach, Copenhagen, DK, 2004. Dissertation (PHD in Informations Studies) - Department of Informations Studies, Royal School of Library and Information Science, Copenhagen, DK, 2004. 399 p.

ESPANHA. Consejo Superior de Investigaciones Científicas. Cybermetrics Lab. Ranking web of world universities 2009. Disponível em: <http://www.webometrics.info/about.html>. Acesso em: 11 jul. 2009.

GOUVEIA, F. C. Estudo Webométrico de Associações de Museus e Centros de Ciência, Rio de Janeiro, 2007. Tese (Doutorado em Química Biológica). Universidade Federal do Rio de Janeiro. 
INGWERSEN, P. The calculation of web impacto factors. Journal of Documentation, London, v. 54, n. 2., 1998. p. 236-43.

SMITH, A. G. A tale of two web spaces: comparing sites using web impact factors. Journal of Documentation, London, v. 55, n. 5, dec. 1999. p. 577-92.

THELWALL, M. Web impact factors and search engine coverage. Journal of Documentation, London, v. 56, n. 2, p. 185-9, 2000.

. Evidence for the existence of geographic trends in university Web site interlinking. Journal of Documentation, London, v. 58, n. 5, p. 563-74, 2002b.

. The top 100 linked-to pages on UK university web sites: high inlink counts are not usually assiciated with quality scholarly content. Journal of Information Science, London, v. 28, n. 6, p. 485-93, 2002a.

. What is this link doing here? Beginning a fine-grained process of identifying reasons for academic hyperlink creation. Information Research, Gothenburg, v. 8, n. 3 apr. 2003.

; SMITH, A. G. A study of the interlinking between Asia-Pacific University Web sites. Scientometrics, v. 55, n. 3, p. 335-48, 2002.

VANTI, N. Application of the Indicators to the Latin-American Academic Sites in Social Sciences. Brazilian Journal of Information Science, Marília, v. 1, n. 2, p. 22-46, 2007.

Da Bibliometria à Webometria: uma exploração conceitual dos mecanismos utilizados para medir o registro da informação e a difusão do conhecimento. Ciência da Informação, Brasília, v. 31, n. 2, p. 152-62, 2002.

Os Links e os Estudos Webométricos. Ciência da Informação, Brasília, v. 34, n. 1, p. 78-88, 2005.

\section{Title}

Mapping federal institutions of higher education of the northeast of Brazil on the web

\section{Abstract}

As part of a larger study concerning the presence of federal institutions of higher education in Brazil on the web, we presents in this work an analysis of universities in the Northeast region of the country through the application of different webometric indicators: size of the site, visibility, web impact factor - FIW, luminosity and the network density. For measuring the size of websites and their visibility in the virtual world, we use the Yahoo search engine. The impact factor is calculated using a formula specially created for this purpose. The luminosity of these sites is calculated with the Xenu Link Steuth program. The density of the network is also obtained by applying a formula. The Ucinet6 program is used to visualize the connections between the sites of universities in the 
region. The results shows that the largest universities and most important are also the ones more linked and the smallest are those that tend to link more, the impact factor do not show significant results when applied to the web environment and density of the network of federal universities in the Northeast of Brazil is very low, which represents a huge untapped potential with regard to the connectivity of these institutions with their peers.

\section{Keywords}

Webometrics. Metric studies of information. Academic web sites Evaluation. FIHE Northeast of Brasil.

\section{Título}

Mapeamiento de las instituciones federales de educación superior de la región noreste de Brasil en la web

\section{Resumen}

Como parte de una investigación más amplia sobre la presencia de las instituciones federales de educación superior brasileñas en la Web, en este trabajo se hace un análisis de las universidades de la región Noreste del país por medio de la aplicación de diferentes indicadores webométricos: tamaño del sitio, visibilidad, factor de impacto Web - FIW, luminosidad y densidad de la red. Para la medición del tamaño de los sitios Web y de su visibilidad en el mundo virtual, se usa el motor de búsqueda Yahoo. El factor de impacto se calcula por medio de una fórmula especialmente diseñada para tal fin. La luminosidad de estos sitios se calcula con el auxilio del programa mapeador Xenu Link Sleuth. La densidad de la red se obtiene también con la aplicación de una fórmula. El programa Ucinet6 se usa para visualizar las conexiones entre los sitios de las universidades de la región. Los resultados demuestran que las universidades más grandes y más importantes son, también, las más accesadas, y las más chicas son las que suelen accesar más, el factor de impacto no presenta resultados significativos cuando es aplicado a sitios Web y la densidad de la red de las universidades federales de la región Noreste del Brasil es muy baja, lo que representa un enorme potencial inexplorado en lo que hace a la conectividad de estas instituciones con sus pares en este ambiente.

\section{Palabras clave}

Webometria. Estudios métricos de la información. Evaluación de sítios académicos. IFES Noreste de Brasil.

Recebido em: 15.03.2010

Aceito em: 15.09 .2010 